\title{
Bridge Numbers of Torus Knots
}

\author{
Jennifer Schultens
}

September 26, 2005

\begin{abstract}
We provide a new self contained proof of the following result of $H$. Schubert: If $K$ is a $(p, q)$-torus knot, then the bridge number of $K$ is $\min (p, q)$.
\end{abstract}

Nearly 50 years ago, Horst Schubert had a premonition of what later became known as JSJ decompositions. He realized that a knot can have only finitely many companion tori. This followed from his work in "Knoten und Vollringe" [1] and "Über eine numerische Knoteninvariante" [2]. In "Über eine numerische Knoteninvariante" [2], he introduced the notion of bridge number for a knot and established a number of properties. Many of these properties are of independent interest. For instance, the fact that a $(p, q)$-torus knot has bridge number $\min \{p, q\}$ is used quite frequently. We here provide a self contained proof of this fact. This proof differs from Schubert's in that it relies on the consideration of Morse functions on $\mathbb{S}^{3}$ whose level sets are spheres (except for the maximum and minimum) and on the foliations they induce on $\mathbb{S}^{3}$. A trick involving symmetry further simplifies the argument. I wish to thank Ray Lickorish and Marty Scharlemann who fostered my interest in Schubert's work and Michel Boileau who suggested that a more contemporary proof of this theorem would be a welcome addition to the existing literature.

In the following $K$ will be a knot in $S^{3}$ and $h: S^{3} \rightarrow \mathbf{R}$ a Morse function with exactly two critical points. This last assumption guarantees that $h$ induces a foliation of $S^{3}$ by spheres, along with one maximum that we denote by $\infty$ and one minimum that we denote by $-\infty$.

Definition 1. If the minima of $\left.h\right|_{K}$ occur below all maxima of $\left.h\right|_{K}$, then we say that $K$ is in bridge position with respect to $h$. The bridge number of $K, b(K)$, is the minimal number of maxima required for $\left.h\right|_{K}$. (Note that this number is independent of whether or not we require $K$ to be in bridge position. Indeed, if $\left.h\right|_{K}$ has $n$ maxima, then the maxima of $\left.h\right|_{K}$ can be raised, and the minima of $\left.h\right|_{K}$ lowered, to obtain a copy of $K$ in bridge position with $n$ maxima.)

Definition 2. Let $T$ be a torus and suppose that $K$ is homotopically nontrivially contained in $T$. Then $T$ is taut with respect to $b(K)$, if the number of critical points of $\left.h\right|_{T}$ is minimal subject to the condition that $\left.h\right|_{K}$ has $b(K)$ maxima.

Definition 3. Consider the singular foliation, $\mathcal{F}_{T}$, of $T$ induced by $\left.h\right|_{T}$. Let $\sigma$ be a leaf corresponding to a saddle singularity. Then $\sigma$ consists of two circles, $s_{1}, s_{2}$, wedged at a point. If either $s_{1}$ or $s_{2}$ is inessential in $T$, then we call $\sigma$ an inessential saddle. Otherwise, $\sigma$ is an essential saddle. 
We first prove a result that extends [3, Lemma 1].

Lemma 1. (The Pop Over Lemma) Let $h, K, T, \mathcal{F}_{T}$ be as above. Suppose that $\mathcal{F}_{T}$ contains inessential saddles. Then there is an isotopy of $T$, that does not raise (but possibly lowers) the number of critical points of $\left.h\right|_{K}$ or of $\left.h\right|_{T}$, after which there is an inessential saddle $\sigma$ in $\mathcal{F}_{T}$ for which the following hold:

1) $s_{1}$ bounds a disk $D_{1} \subset T$ such that $\mathcal{F}_{T}$ restricted to $D_{1}$ contains only disjoint circles and one maximum or minimum; and

2) $D_{1} \cap K=\emptyset$; and

3) for $L$ the level surface of $h$ containing $\sigma, D_{1}$ cobounds a 3-ball $B$ with a disk $\tilde{D}_{1} \subset L$, such that $B$ does not contain $\infty$ or $-\infty$, and such that $s_{2}$ does not meet $B$ (i.e., such that $s_{2}$ lies in $L-\tilde{D}_{1}$ ).

Proof: To satisfy the first condition, we must merely choose $\sigma$ to be an inessential saddle in $\mathcal{F}_{T}$ that is innermost in $T$.

To see that the second condition is satisfied, consider the disk $D_{1}$ in $T$ bounded by $s_{1}$. It contains exactly one extreme point. We may assume that this extreme point is a maximum. All other level sets are circles. Here $K \cap D_{1}$ can't contain any closed curves, so it consists of a collection of arcs. By transversality, the endpoints of the arcs do not lie on $s$. We may assume that each arc in this collection has exactly one maximum; indeed, if this is not the case, then we can replace the collection of arcs with an isotopic collection in which each arc has exactly one maximum and thereby lower the number of maxima of $\left.h\right|_{K}$. Now an outermost arc from this collection can be isotoped out of $D_{1}$ in such a way that the number of critical points of $\left.h\right|_{K}$ remains unchanged.

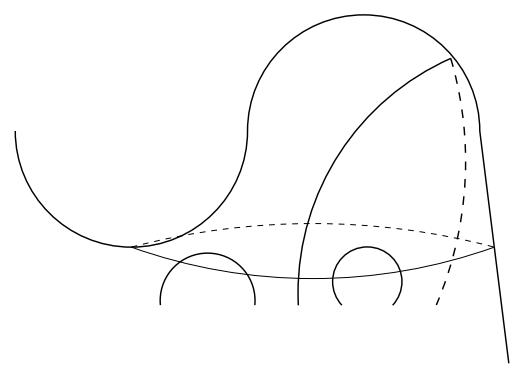

Figure 1: Before isotoping the arcs

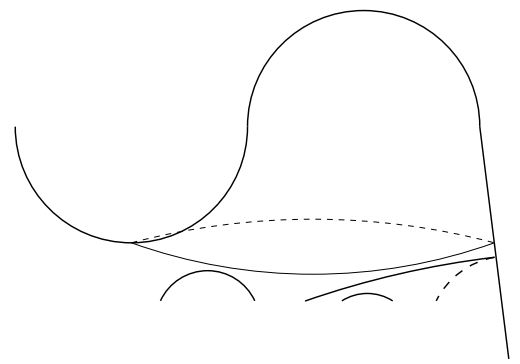

Figure 2: After isotoping the arcs 
Verifying the third condition requires a more intricate argument. Here $L-\partial D_{1}$ consists of two disks, $\hat{D}_{1}$ and $\hat{D}_{2}$. Together with $D_{1}$, both $\hat{D}_{1}$ and $\hat{D}_{2}$ cobound 3-balls $\hat{B}_{1}, \hat{B}_{2}$, respectively. One of these 3 -balls, say $\hat{B}_{2}$, contains either $\infty$ or $-\infty$ and the other contains neither. Since we are assuming that the critical point of $D_{1}$ is a maximum, $\hat{B}_{2}$ contains $\infty$.

If $s_{2} \subset \hat{D}_{2}$, we may take $B=\hat{B}_{1}$, so suppose $s_{2} \subset \hat{D}_{1}$. In this case, consider a monotone arc $\alpha$ beginning at the maximum of $D_{1}$, passing only through maxima of $T$ and ending at $\infty$. Note that by transversality, $K$ does not meet any maxima of $T$, so $\alpha$ can be taken to be disjoint from $K$. Let $a_{1}, \ldots, a_{n}$ be the points at which $\alpha$ meets $T$, with $a_{n}$ the highest such point.

Let $\beta$ be the subarc of $\alpha$ between $a_{n}$ and $\infty$ and let $C^{\prime}$ be a collar neighborhood of $\beta$. After a small isotopy, $T \cap C^{\prime}$ consists of a small disk $D=a_{n} \times \operatorname{disk} \subset T$. Let $C^{\prime \prime}$ be a small 3-ball centered at $\infty$ that is disjoint from $T$. Set $C=C^{\prime} \cup C^{\prime \prime}$ and consider $T^{\prime}=(T-D) \cup(\partial C-D)$. This describes an isotopy of $T$ that replaces $\hat{B}_{1}$ by $\hat{B}_{1} \cup C$ and replaces $\hat{B}_{2}$ by $\hat{B}_{2}-C$. After a small tilt which turns $\left.h\right|_{T^{\prime}}$ into a Morse function, the maximum $a_{n}$ of $\left.h\right|_{T}$ has turned into a maximum of $\left.h\right|_{T^{\prime}}$ at a higher level. No critical points need have been introduced for $h_{K}$ and the number of critical points of $\left.h\right|_{T^{\prime}}$ is the same as that of $\left.h\right|_{T}$.

By induction, we may assume that $\alpha$ is disjoint from $T$ except at its initial point. Then if $s_{2} \subset \hat{D}_{1}$, this same construction using $\beta=\alpha$ describes an isotopy of $T$ augmenting $\hat{B}_{1}$ to contain $\infty$ and shrinking $\hat{B}_{2}$ to exclude $\infty$ without introducing any critical points of $\left.h\right|_{K}$ or $\left.h\right|_{T}$. We may then choose $B$ to be the shrunk version of $\hat{B}_{2}$. q.e.d.

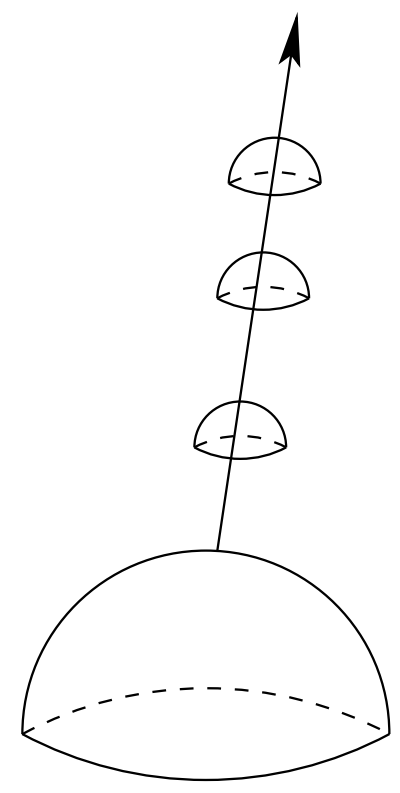

Figure 3: The arc $\alpha$

The following Lemma is [3, Lemma 2]. We include it here for completeness.

Lemma 2. (The Pop Out Lemma) Let $h, K, T, \mathcal{F}_{T}$ be as above. If $T$ is taut with respect to $b(K)$, then there are no inessential saddles in $\mathcal{F}_{T}$. 
Proof: Suppose there are inessential saddles. Alter $T$ as in Lemma 1 so that there is an inessential saddle $\sigma$ satisfying the conclusions of Lemma 1. We may assume that $D_{1}$ contains a maximum and lies above $L$. (The other case is analogous.) Here $(K \cup T) \cap \operatorname{int}(B)$ can be shrunk horizontally and lowered via an isotopy to lie just below $\tilde{D}_{1}$ (and above any critical points of $\left.h\right|_{K}$ or $\left.h\right|_{T}$ below $\tilde{D}_{1}$ ). This does not change the nature or number of critical points of $\left.h\right|_{K}$ or $\left.h\right|_{T}$.

Now $D_{1} \subset T$ can be replaced by $\tilde{D}_{1}$ to obtain $\tilde{T}$. After a small tilt of $\tilde{T},\left.h\right|_{\tilde{T}}$ is a Morse function. Furthermore, $\tilde{T}$ is isotopic to $T$. Yet $\left.h\right|_{\tilde{T}}$ has two fewer critical points than $h_{T}$. (A maximum and an inessential saddle have been cancelled). This contradicts the assumption that $T$ is taut with respect to $b(K)$. q.e.d.

Remark 4. Consider a bicollar $N$ of an essential saddle $\sigma$ in $T$. It has three boundary components, $c_{1}, c_{2}, c_{3}$. Here $c_{i}$ is parallel to $s_{i}$ for $i=1,2$. Furthermore, $\chi(N)=-1$, so $\chi(T-N)=1$. Since $N$, and hence also $T-N$, has three boundary components, $T-N$ consists of one annulus and one disk. It follows that $c_{3}$ bounds a disk $D_{3}$. In particular, $c_{1}$ and $c_{2}$ are parallel on $T$.

Theorem 1. Suppose $K$ is a $(p, q)$-torus knot. Then $b(K)=\min \{p, q\}$.

Proof: We may assume that $T$ is taut with respect to $b(K)$ and let $\sigma$ be the highest saddle in $\mathcal{F}_{T}$. By Lemma $2, \sigma$ is essential. Let $c_{1}, c_{2}$, be as above and assume that $c_{1}$ and $c_{2}$ are contained in the same level surface $L$. Let $\hat{D}_{1}, \hat{D}_{2}$ be the (disjoint) disks

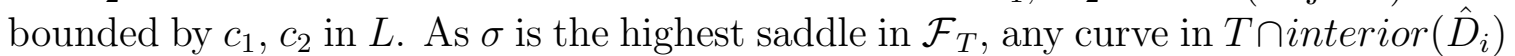
bounds a disk lying above $L$. This implies that $\hat{D}_{i}$ is isotopic to a disk whose interior is disjoint from $T$. Since $\partial \hat{D}_{i}$ is an essential curve in $T$ that bounds a disk in $\mathbb{S}^{3}-T$ it is either a meridian or a longitude of $T$. Moreover, if $\partial \hat{D}_{1}$ is a meridian, then so are $\partial \hat{D}_{2}, c_{1}$ and $c_{2}$, and if $\partial \hat{D}_{1}$ is a longitude, then so are $\partial \hat{D}_{2}, c_{1}$ and $c_{2}$. In either case, $K \cap c_{i} \geq \min \{p, q\}$ for $i=1,2$. Now let $N, D_{3}$ be as in Remark 4 and consider $K \cap\left(N \cup D_{3}\right)$. In $N \cup D_{3}$, the points $K \cap c_{i} \geq \min \{p, q\}$ for $i=1,2$ are connected by arcs. Since all these points occur at the same level each such arc contains at least one maximum. It follows that $b(K) \geq \min \{p, q\}$.

To see that $b(K) \leq \min \{p, q\}$, consider Figure 4. q.e.d.

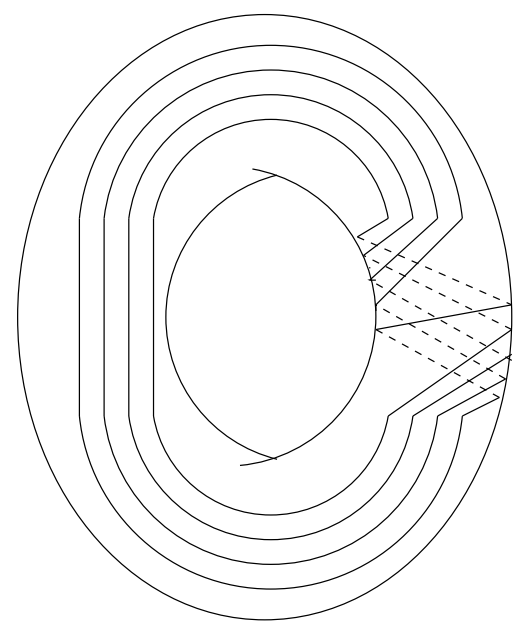

Figure 4: A realization of the bridge number of the $(5,4)$-torus knot 


\section{References}

[1] Schubert, Horst: "Knoten und Vollringe." Acta Math. 90 (1953), 131-286.

[2] Schubert, Horst: "Über eine numerische Knoteninvariante." Math. Z. 61 (1954) $245-288$

[3] Schultens, Jennifer: "Additivity of bridge numbers of knots." Math. Proc. Cambridge Philos. Soc. 135 (2003), no. 3, 539-544.

Department of Mathematics

1 Shields Avenue

University of California, Davis

Davis, CA 95616

USA 